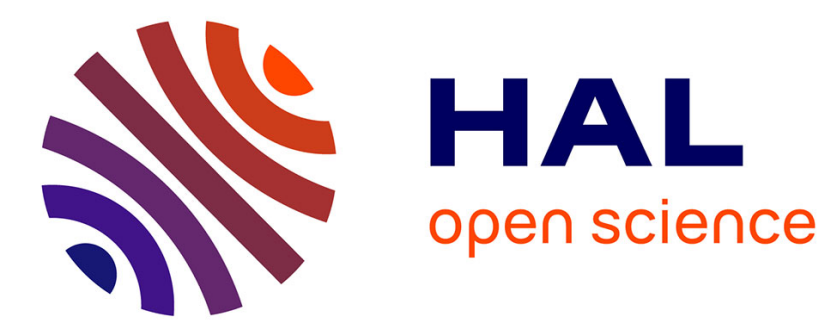

\title{
Production et étude d'un faisceau moléculaire super sonique à symétrie plane
}

Jean-Pierre Treguier, Monique Hagene, Danièle Pasquerault

\section{To cite this version:}

Jean-Pierre Treguier, Monique Hagene, Danièle Pasquerault. Production et étude d'un faisceau moléculaire super sonique à symétrie plane. Revue de Physique Appliquée, 1970, 5 (2), pp.343-350. 10.1051/rphysap:0197000502034300 . jpa-00243400

\section{HAL Id: jpa-00243400 https://hal.science/jpa-00243400}

Submitted on 1 Jan 1970

HAL is a multi-disciplinary open access archive for the deposit and dissemination of scientific research documents, whether they are published or not. The documents may come from teaching and research institutions in France or abroad, or from public or private research centers.
L'archive ouverte pluridisciplinaire HAL, est destinée au dépôt et à la diffusion de documents scientifiques de niveau recherche, publiés ou non, émanant des établissements d'enseignement et de recherche français ou étrangers, des laboratoires publics ou privés. 


\title{
PRODUCTION ET ÉTUDE D'UN FAISCEAU MOLÉCULAIRE SUPERSONIQUE A SYMÉTRIE PLANE
}

\author{
par Jean-Pierre TREGUIER, Monique HAGENE et Danièle PASQUERAULT \\ Laboratoire de Physique Moléculaire, Faculté des Sciences, 35, Rennes
}

(Reçu le 30 juin 1969, revisé le 16 janvier 1970)

\begin{abstract}
Résumé. - On décrit et étudie un appareil de production de faisceau supersonique de section rectangulaire. Le nombre de Mach $M$ dans le flux continu est déterminé par une méthode semiempirique, semi-théorique. On montre que $M$ tend vers une limite. Une étude expérimentale de l'intensité et de sa distribution dans les directions perpendiculaires à l'axe du faisceau, a été faite en fonction de la pression amont buse $p_{0}$ et de la distance buse-éplucheur $b$. L'intensité obtenue dans les conditions optimales est de $2,1 \times 10^{18} \mathrm{~mol}$. srd $^{-1} . \mathrm{s}^{-1}$ pour l'azote. Ceci représente $15 \%$ de la valeur théorique calculée. On donne les performances par rapport à un faisceau de four et par rapport à un faisceau supersonique de révolution.
\end{abstract}

Abstract. - We describe and study a supersonic beam apparatus with plane symmetry. The Mach number $M$ in the continuum flow is determined by a half-empirical, half-theoretical method. We show that $M$ tends to a limit. An experimental study of the intensity and of the intensity distributions in the directions perpendicular to the beam axis, was made versus the upstream nozzle pressure $p_{0}$ and versus nozzle-skimmer distance $b$. The intensity obtained in the optimal conditions is $2.1 \times 10^{18} \mathrm{~mol}$. $\mathrm{srd}^{-1} . \mathrm{s}^{-1}$. for nitrogen. This represents $15 \%$ of the calculated theoretical value. We give the performances with respect to an oven beam and supersonic beam with axial symmetry.

Introduction. - Les faisceaux d'origine effusive utilisés depuis 1914 par beaucoup de chercheurs se sont montrés insuffisants pour de nombreuses applications à cause de leur faible intensité entraînant des difficultés de détection. En 1951, Kantrowitz et Grey [1] suggérèrent de remplacer le gaz du four par un écoulement supersonique en utilisant une buse. C'est seulement en 1954 que Becker et ses collaborateurs [2] ont mis en évidence expérimentalement les trois avantages des faisceaux supersoniques par rapport à ceux de four : intensité plus élevée, distribution de vitesses plus étroite et énergie supérieure. Les divers dispositifs réalisés jusqu'à présent produisent des faisceaux à symétrie axiale; seuls Kistiakowsky et Slichter [3] ont essayé, sans succès, d'obtenir un faisceau plan. Une symétrie plane étant très intéressante pour des applications dans de nombreux domaines, en particulier dans l'étude des collisions, nous nous sommes attachés à obtenir un faisceau d'origine supersonique de section rectangulaire.

1. Principe. - Le gaz au repos d'un système classique de four est remplacé par un écoulement supersonique obtenu à partir d'une buse (Fig. 1). Dans ces conditions, la paroi de la fente effusive est un obstacle dans la détente supersonique et provoque une onde de choc "détachée », qui détruit alors cette détente. Pour éviter ce phénomène, on remplace la paroi précédente par une paroi en forme de cône d'angle bien déterminé : nous appellerons «éplucheur » cet élé-
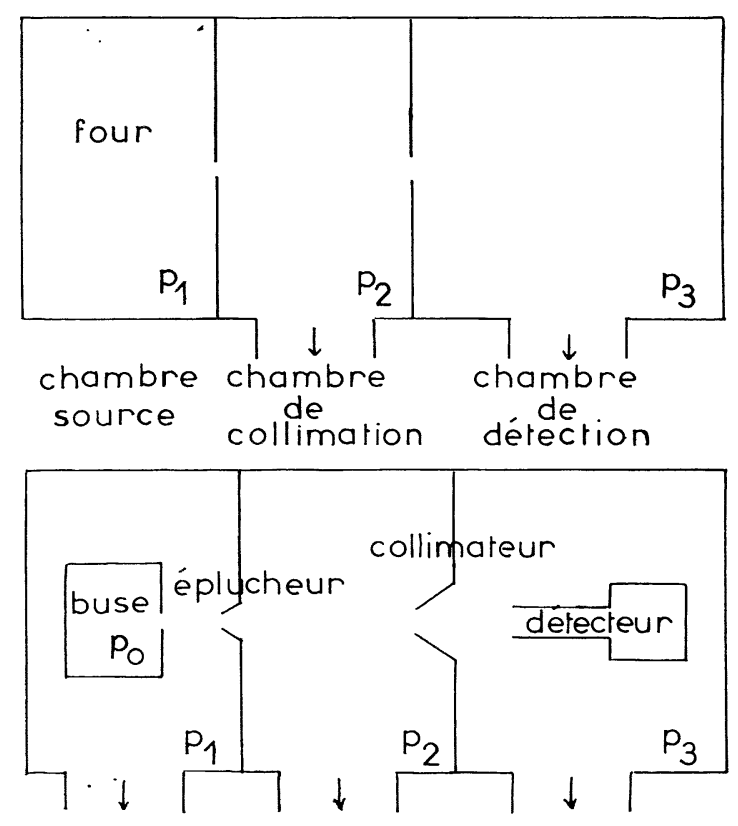

FIG. 1. - Schéma de principe de la production : $a$ ) d'un faisceau de four ; $b$ ) d'un faisceau supersonique.

ment (Fig. 2). L'éplucheur extrait le centre du flux. En aval de l'éplucheur le flux devient moléculaire après avoir subi une transition [1] [4].

La vitesse d'entraînement $U$ des molécules et le refroidissement du flux, obtenus au cours de la détente, 


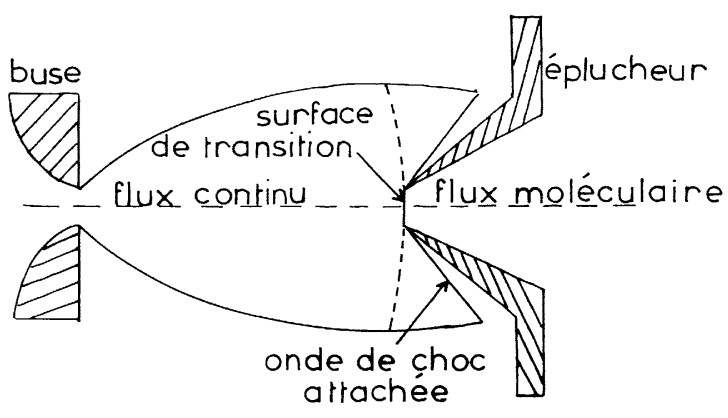

FIG. 2. - Schéma d'une détente d'après Kantrowitz et Grey.

apportent trois améliorations par rapport à un faisceau de four :

- le débit est plus important au premier orifice, la vitesse moyenne d'agitation thermique dans la direction axiale, $u$, étant petite par rapport à $U$ (Fig. 3) ;

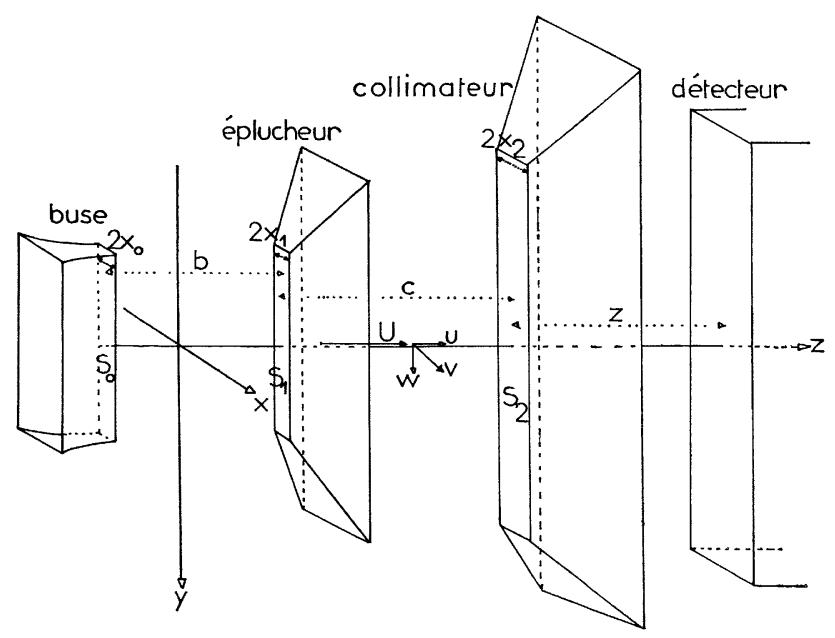

FIG. 3. - Disposition des éléments dans l'espace $O x y z$.

- un nombre plus grand de molécules persiste sur l'axe en aval de l'éplucheur, la vitesse moyenne d'agitation thermique dans la direction radiale étant petite par rapport à $U$;

- les molécules ont une distribution de vitesse plus étroite, le gaz étant refroidi au cours de la détente.

Ces trois effets se superposent et entraînent un gain théorique en intensité de l'ordre de 50 à 200.

2. Théorie. - 2.1 InTEnsité DU faisceau. - La première évaluation de l'intensité du faisceau, valable quelle que soit la géométrie des éléments de définition, fut faite par Kantrowitz et Grey. Elle repose sur les hypothèses suivantes :

- le flux est continu entre la buse et l'éplucheur ;

- la détente à partir de la chambre buse jusqu'à l'éplucheur est isentropique ;

- $\gamma$, rapport des chaleurs spécifiques à pression constante et à volume constant, est constant ;
- le flux à l'entrée de l'éplucheur est supersonique et non perturbé ;

- en aval de la fente de l'éplucheur, il n'y a plus de collisions moléculaires.

Cette théorie suppose qu'il y a une transition du flux continu en flux moléculaire, qui a lieu à l'éplucheur. Cette théorie fait intervenir le nombre de Mach à ce niveau. A partir de l'éplucheur, la vitesse $U$ et la température $T$ sont alors constantes ainsi que le nombre de Mach $M_{t}$ :

$$
M_{t}=\frac{U}{\sqrt{\frac{\gamma R T}{m}}}
$$

où $R$ est la constante des gaz parfaits et $m$ la masse moléculaire. Dans une première approximation Kantrowitz et Grey négligent $u$ devant $U$ et supposent que l'orifice de collimation est petit. Le gain en intensité est alors $(\gamma / 2) M_{t}^{2}$ par rapport à un faisceau de four si on considère que les dimensions de la fente effusive et la densité en amont de cette fente sont les mêmes pour le faisceau supersonique et le faisceau de four.

Anderson, Andres et Fenn [4], tenant compte des résultats expérimentaux obtenus avec des faisceaux de révolution ont montré que cette transition peut avoir lieu en amont de l'éplucheur et n'ont pas négligé $u$. Ils trouvent alors une intensité en aval du collimateur :

$$
\begin{aligned}
& I_{2}=\frac{n_{1} S_{1} a_{0} M_{t}}{2 \pi} \frac{\left[\gamma M_{t}^{2}+3\right]}{\left[1+\frac{\gamma-1}{2} M_{t}^{2}\right]^{1 / 2}} \times \\
& \quad \times\left[\frac{b}{b}\right]^{2} \frac{S_{2}}{c^{2}} \mathrm{~mol} . \mathrm{s}^{-1}
\end{aligned}
$$

où $n_{1}$ est la densité à l'éplucheur, $a_{0}$ la vitesse du son en amont de la buse, $b_{t}$ la distance de la buse à la surface de transition et $S_{1}, S_{2}, b$ et $c$ sont définis sur la figure 3.

Or

$$
n_{1}\left[\frac{b}{b_{t}}\right]^{2}=n_{t}=n_{0}\left[1+\frac{\gamma-1}{2} M_{t}^{2}\right]^{1 /(\gamma-1)}
$$

où $n_{0}$ est la densité en amont de la buse et $n_{t}$ la densité à la surface de transition. D'où

$$
\begin{array}{r}
I_{2}=\frac{n_{0} S_{1} a_{0} M_{t}}{2 \pi} \frac{\left[\gamma M_{t}^{2}+3\right]}{\left[1+\frac{\gamma-1}{2} M_{t}^{2}\right]^{(\gamma+1) / 2(\gamma-1)}} \times \\
\times \frac{S_{2}}{c^{2}} \text { mol. s }{ }^{-1}
\end{array}
$$

qui ne dépend que de $M_{t}$.

2.2 Calcul du Nombre de MaCh $M$ EN tout PoINT DU FluX CONTINU. - Bier et Schmidt [5] ont donné, pour déterminer ce nombre de Mach $M$, une méthode semi-théorique, semi-empirique dont les résultats 
concordent, pour les systèmes de révolution, avec les résultats théoriques de Owen et Thornhill [6].

Aucun calcul de $M$ n'ayant été fait pour des buses de section rectangulaire en régime raréfié, nous avons appliqué à ce cas la méthode de Bier et Schmidt.

2.2.1 Méthode de Bier et Schmidt. - Le nombre de Mach $M$ en un point du flux est relié à la pression $p$ en ce point par :

$$
M^{2}=\frac{2}{\gamma-1}\left[\left(\frac{p_{0}}{p}\right)^{(\gamma-1) / \gamma}-1\right] .
$$

Les conditions en amont de la tuyère $\left(p_{0}, T_{0}, n_{0}\right)$ et les conditions en amont $\left(p^{\prime}, T^{\prime}, n^{\prime}\right)$ et en aval $\left(p_{M}, T_{M}\right.$, $n_{M}$ ) du disque de Mach éventuel sont reliées entre elles par :

$$
\begin{aligned}
\frac{n_{M}}{n^{\prime}} & =\frac{\gamma+1}{\gamma-1}\left[1-\frac{T^{\prime}}{T_{0}}\right] \\
\frac{T_{M}}{T_{0}} & =\frac{4 \gamma}{(\gamma+1)^{2}}\left[1-\frac{(\gamma-1)^{2}}{4 \gamma} \cdot \frac{T^{\prime}}{T_{0}}\left(1-\frac{T^{\prime}}{T_{0}}\right)^{-1}\right] \\
\frac{n_{0}}{n^{\prime}} & =\frac{p_{0} T_{M}}{p_{M} T_{0}} \frac{n_{M}}{n^{\prime}} .
\end{aligned}
$$

Ces trois relations donnent, en tenant compte de l'hypothèse de Adamson et Nicholls [7] :

$$
p_{M}=p_{1}=\text { pression résiduelle en aval de la buse }
$$
et de l'approximation supersonique $T^{\prime} / T_{0} \ll 1$ :

$$
\frac{p_{0}}{p^{\prime}}=\left[\frac{4 \gamma}{\gamma^{2}-1} \cdot \frac{p_{0}}{p_{1}}\right]^{\gamma}
$$

$M^{\prime}$ en amont du disque de Mach est alors fonction du rapport $p_{0} / p_{1}$ :

$$
M^{\prime 2}=\frac{2}{\gamma-1}\left[\left(\frac{4 \gamma}{\gamma^{2}-1} \cdot \frac{p_{0}}{p_{1}}\right)^{\gamma-1}-1\right] .
$$

2.2.2 Application aux buses de section rectangulaire. - Bier et Schmidt ont déterminé, par une méthode strioscopique, que la position $b^{\prime}$ de l'onde de choc, rapportée à la dimension caractéristique $2 x_{0}$ de la buse, est fonction du rapport $p_{0} / p_{1}$ et ils ont donné ces fonctions pour les systèmes de révolution et pour les systèmes à symétrie plane. Mais ces auteurs n'ont tracé les courbes donnant $M^{\prime}$ en fonction de $b^{\prime} / 2 x_{0}$ que pour les systèmes de révolution. Nous avons poursuivi leur étude en déterminant $M^{\prime}$ en fonction de $b^{\prime} / 2 x_{0}$ pour les systèmes à symétrie plane pour $\gamma=5 / 3,7 / 5,9 / 7$. Comme le nombre de Mach au point considéré est indépendant de toute onde de choc ou «disque de Mach » qui apparaît juste en aval de ce point, $M^{\prime}=f\left(b^{\prime} / 2 x_{0}\right)$ représente donc aussi le nombre de Mach $M$ en tout point situé à la distance $b_{z}$ de la buse (Fig. 4). Nous avons ainsi obtenu la relation empirique suivante :

$$
M^{2}=\frac{2}{\gamma-1}\left[\left(\frac{4 \gamma}{\gamma^{2}-1}\right)^{\gamma-1}\left(0,68 \frac{b_{z}}{2 x_{0}}\right)^{(\gamma-1) / 0,7}-1\right] .
$$

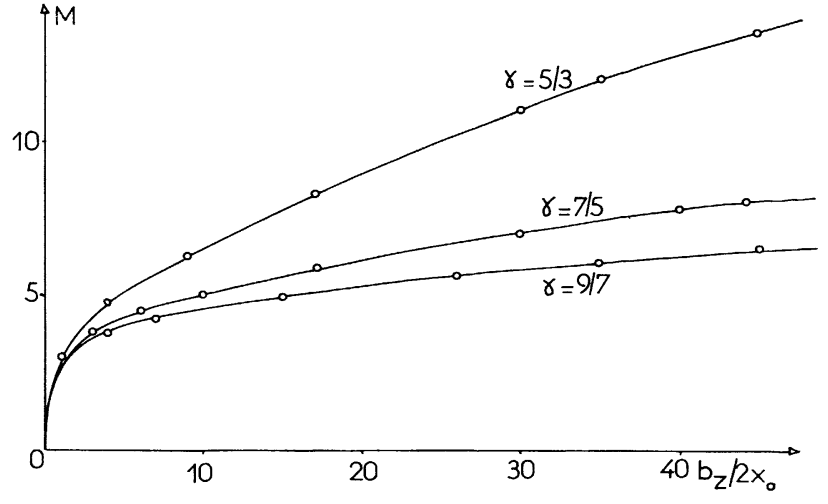

FIg. 4. - Nombre de Mach $M$ en tout point dans le flux continu, obtenu à partir des études strioscopiques de Bier et Schmidt.

2.3 PhÉNOMÈne De RARÉFACTION et CALCUl DE $M_{t}$. - D'après la relation (3) $M$ peut croître constamment mais, aux basses pressions $p_{0}$, la densité du gaz dans le flux peut devenir si faible qu'aucune collision ne se produit. Il y a un effet de raréfaction et $M$ atteint une limite $M_{t}$ (on dit qu'il y a " gelage » de $M$ ). Cet effet est lié à la quantité de gaz sortant de la buse et par conséquent au nombre de Knudsen $K n_{0}$ :

$$
K n_{0}=\frac{\lambda_{0}}{2 x_{0}}
$$

où $\lambda_{0}$ est le libre parcours moyen en amont de la buse.

La valeur $M_{t}$ a été déterminée, pour une buse convergente de révolution, par Oman [8] qui a montré que pour $b_{z}$ donné, la dérivée de $M$ par rapport à $b_{z}$ ne peut être supérieure à une valeur $\left[\mathrm{d} M / \mathrm{d} b_{z}\right]_{\max }$ donnée par:

$$
\left[\frac{\mathrm{d} M}{\mathrm{~d} b_{z}}\right]_{\max }=\left(\frac{8}{\pi \gamma}\right)^{1 / 2} \cdot \varepsilon \lambda^{-1}
$$

où $\varepsilon$ est un paramètre dépendant de la section efficace de collision et $\lambda$ le libre parcours moyen à la distance $b_{z}$.

A partir de cette relation, nous obtenons pour $\gamma=5 / 3$

$$
\left[\frac{\mathrm{d} M}{\mathrm{~d}\left(\frac{b_{z}}{2 x_{0}}\right)}\right]_{\max }=0,575 \cdot \varepsilon \cdot K n_{0}^{-1}\left(\frac{b_{z}}{2 x_{0}}\right)^{-3 / 2} .
$$

La figure 5 représente cette fonction pour une valeur de $\varepsilon K n_{0}{ }^{-1}$ ainsi que la fonction prévue par la théorie du flux continu :

$$
\left[\frac{\mathrm{d} M}{\mathrm{~d}\left(\frac{b_{z}}{2 x_{0}}\right)}\right]_{\mathrm{cont}}=1,12\left(\frac{b_{z}}{2 x_{0}}\right)^{-1 / 2} .
$$




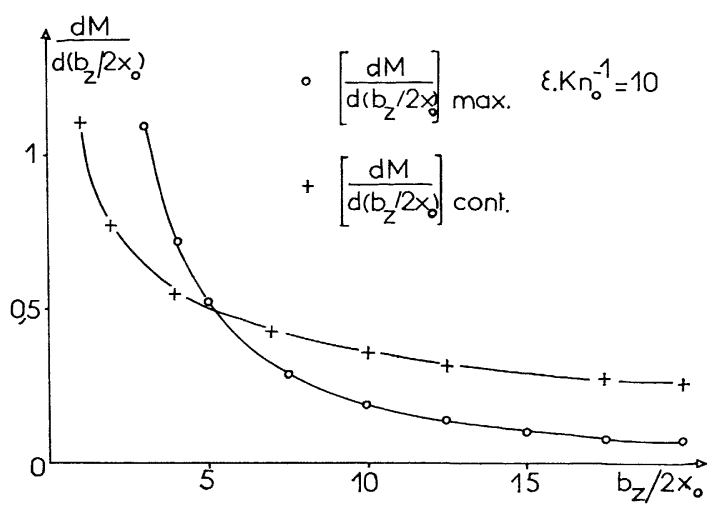

Fig. 5. - Dérivées du nombre de Mach par rapport à $b_{z} / 2 x_{0}$ prévues par la formule de Oman et par la théorie du flux continu.

Cette dernière courbe est valable jusqu'à son point d'intersection avec la courbe

$$
\left[\frac{\mathrm{d} M}{\mathrm{~d}\left(\frac{b_{z}}{2 x_{0}}\right)}\right]_{\max }, \quad\left[\frac{\mathrm{d} M}{\mathrm{~d}\left(\frac{b_{z}}{2 x_{0}}\right)}\right]_{\mathrm{cont}}
$$

lui étant toujours inférieure. Comme à partir de ce point d'intersection

$$
\left[\frac{\mathrm{d} M}{\mathrm{~d}\left(\frac{b_{z}}{2 x_{0}}\right)}\right]_{\max }
$$

tend rapidement vers zéro, en première approximation on peut considérer que le nombre de Mach $M$ a déjà atteint sa limite $M_{t}$ en ce point. Comme en ce point

$$
\left[\frac{\mathrm{d} M}{\mathrm{~d}\left(\frac{b_{z}}{2 x_{0}}\right)}\right]_{\max }=\left[\frac{\mathrm{d} M}{\mathrm{~d}\left(\frac{b_{z}}{2 x_{0}}\right)}\right]_{\mathrm{cont}}
$$

on trouve pour $M_{t}$ une expression générale de la forme :

$$
M_{t}=A \cdot K n_{0}^{\alpha}
$$

$A$ dépendant de $\varepsilon$ et $\alpha$ de $\gamma$.

3. Description du montage (Fig. 1) [9]. - La buse de section rectangulaire $(0,1 \times 5 \mathrm{~mm})$ est convergente dans le plan de la figure 1 et à parois parallèles dans le plan perpendiculaire. Elle est située à l'extrémité du tube d'arrivée du gaz; l'ensemble coulisse sur des joints en élastomère de façon à faire varier la distance buse-éplucheur de 0 à $30 \mathrm{~mm}$.

L'éplucheur sépare la chambre-source de la chambre de collimation. Sa fente a une section de $0,1 \times 5 \mathrm{~mm}$. Il est en forme de toit à 4 pans (Fig. 3). Les pans opposés 2 à 2 forment les angles extérieur $2 \theta_{\mathrm{e}}$ et intérieur $2 \theta_{\mathrm{i}} .2 \theta_{\mathrm{e}}$ a été déterminé à partir des relations de la thermodynamique du flux continu de façon à éviter la formation d'une onde de choc détachée en amont de l'éplucheur [1]. L'angle intérieur $2 \theta_{\mathrm{i}}$ est déterminé de façon à éviter les collisions des, molécules du faisceau avec les parois internes de l'éplucheur, ce qui est réalisé [1] si :

$$
\sin \theta_{i}>\frac{2}{M} \sqrt{\frac{2}{\pi \gamma}} .
$$

Nous avons tracé les limites supérieure de $\theta_{\mathrm{e}}$ et inférieure de $\theta_{\mathrm{i}}$ en fonction du nombre de Mach pour $\gamma=5 / 3$ (Fig. 6). En considérant que le nombre de Mach sera toujours supérieur à 4 et en tenant compte des possibilités de réalisation nous avons choisi $\theta_{\mathrm{e}}=31^{\circ}$ et $\theta_{\mathrm{i}}=19^{\circ}$. L'éplucheur en acier inoxydable est constitué de deux pièces travaillées séparément, polies optiquement puis frettées.

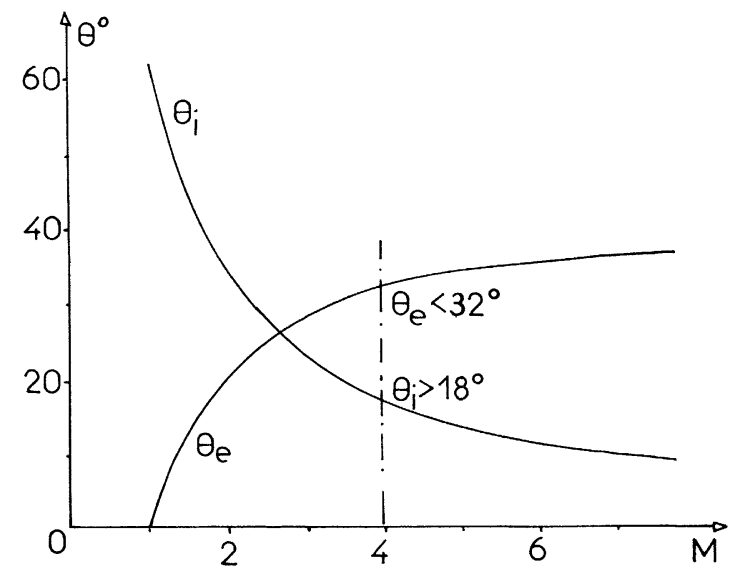

FIG. 6. - Limites supérieure de l'angle $\theta_{\mathrm{e}}$ et inférieure de $\theta_{\mathrm{i}}$ en fonction du nombre de Mach pour $\gamma=5 / 3$.

Le collimateur sépare la chambre de détection de la chambre d'étude. Il a même forme que l'éplucheur et sa fente a une section de $0,2 \times 10 \mathrm{~mm}$. La distance $c$ entre l'ensemble buse-éplucheur et le collimateur varie entre 20 et $50 \mathrm{~mm}$.

Les pressions $p_{0}$ en amont de la buse sont mesurées dans le domaine de 0 à 10 torr à l'aide d'une jauge de Pirani étalonnée pour les différents gaz et dans le domaine de 10 à 100 torr par un manomètre métallique de Bourdon.

L'ensemble du montage est en acier inoxydable et comprend les trois chambres suivantes :

- La chambre source dans laquelle la pression $p_{1}$, mesurée à l'aide d'une jauge de Pirani, varie de $2 \times 10^{-3}$ à $5 \times 10^{-2}$ torr suivant les conditions de fonctionnement, est évacuée par un booster Edwards de $900 \mathrm{ls}^{-1}$. Un piège en cuivre cylindrique et axial, muni de trous pour augmenter la conductance, refroidi à l'air liquide, sert de cryopompe.

- La chambre de collimation reliée directement à une pompe à diffusion d'huile Edwards de $900 \mathrm{ls}^{-1}$ munie d'un baffle, comporte un piège à air liquide de même conception que le précédent. La pression $p_{2}$ mesurée par une jauge à ionisation, varie de $2 \times 10^{-7}$ torr (avec cryopompage) à $10^{-5}$ torr.

- Dans la chambre de détection, reliée directement à une pompe à diffusion d'huile Edwards de $3000 \mathrm{ls}^{-1}$, 
munie d'un baffle à chevrons, la pression résiduelle $p_{3}$, mesurée à l'aide d'une jauge à ionisation, reste peu différente de $10^{-6}$ torr. Cette chambre contient le système de détection du faisceau qui est constitué d'une jauge à ionisation munie d'un tube à compression de longueur $11 \mathrm{~cm}$ et de section $0,6 \times 27 \mathrm{~mm}$. Le système peut subir une translation sous vide commandée de l'extérieur dans les trois directions sur une distance de $30 \mathrm{~cm}$ dans la direction $z$ de propagation du faisceau et de $8 \mathrm{~cm}$ dans les deux directions perpendiculaires $x$ et $y$.

4. Résultats expérimentaux. - -4.1 VARIATION DE L'INTENSITÉ EN FONCTION DE $b$ ET $p_{0}$ POUR L'AZOTE. -La relation (1) montre que l'intensité dépend de la distance $b$ et de $p_{0}$. Nous avons fait une étude de $I=f(b) p_{0}$ pour différents gaz. Les courbes ont toutes la même allure que celles représentées sur la figure 7 pour l'azote.

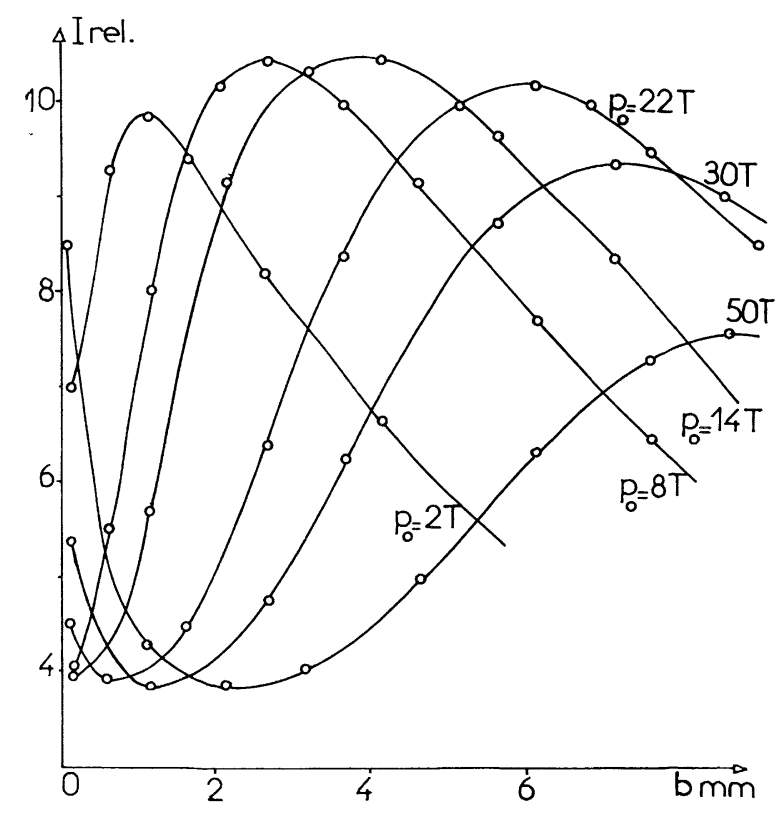

FIG. 7. - Intensité relative du faisceau, pour l'azote, en fonction de la distance $b$ pour différentes valeurs de $p_{0}(z=9 \mathrm{~cm}$, $c=28 \mathrm{~mm}$ ).

4.1.1 Etude des différents domaines des courbes. En général chaque courbe présente trois domaines distincts (I, II et III) (Fig. 8) que nous étudierons successivement.

4.1.1.1 Etude du domaine I. - L'intensité décroît jusqu'à un minimum. Une étude en fonction de la pression $p_{1}$ (Fig. 8) nous a montré que la pente de la décroissance, la position et l'intensité du minimum sont indépendantes de la pression $p_{1}$. Ceci nous a permis de considérer, comme d'autres auteurs [10], que le flux est continu dans ce domaine. Il faut remarquer que ce domaine est réduit à la position $b$ minimale pour les petites valeurs de la pression $p_{0}$.

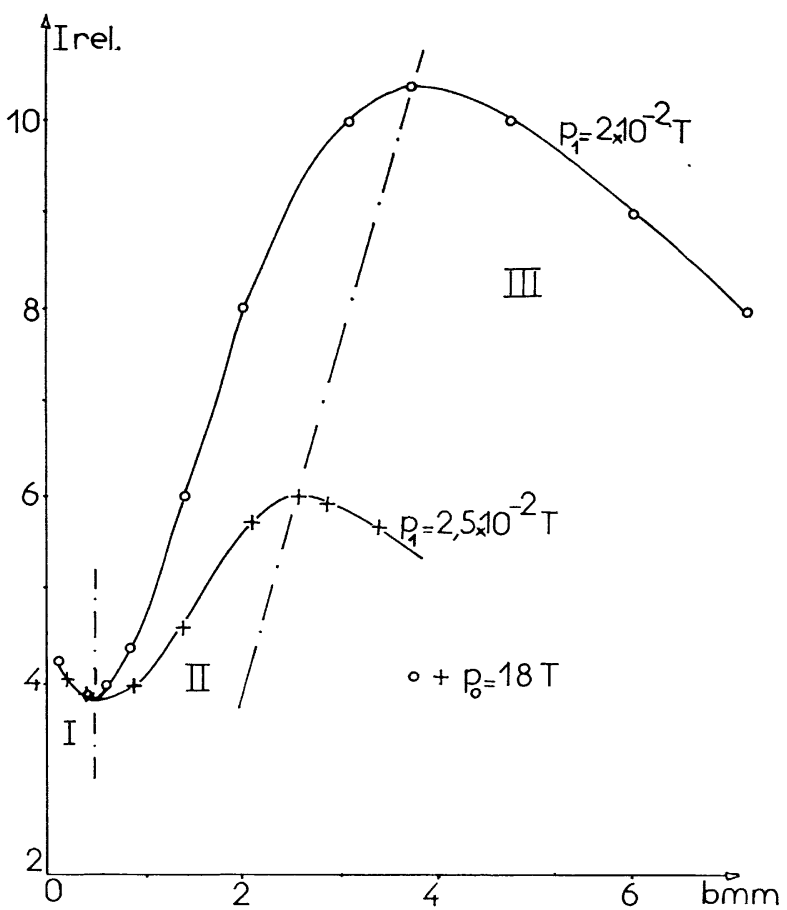

Fig. 8. - Influence de la pression $p_{1}$ et limites des domaines I, II, III pour l'azote $(z=9 \mathrm{~cm}, c=28 \mathrm{~mm})$.

4.1.1.2 Etude du domaine II. - L'intensité croît jusqu'à un maximum. A $p_{0}$ donné, ce maximum apparaît d'autant plus rapidement que $p_{1}$ est plus grand : les molécules du gaz résiduel perturbent davantage le faisceau. Nous pensons que le flux est alors moléculaire à partir du minimum.

D'après la relation (2), $M$ étant alors constant, l'intensité devrait être constante à $p_{0}$ donné. L'augmentation de l'intensité, que nous avons constatée, s'explique par une diminution des perturbations au niveau de l'éplucheur. Par analogie avec un faisceau de four $(K n>1)$ il faut que $K n / M$ soit supérieur à 1 (Kn étant le nombre de Knudsen respectivement à la fente effusive et à l'éplucheur). Ceci sera d'autant mieux vérifié que l'on s'éloigne du minimum.

4.1.1.3 Etude du domaine III. - Quand $K n / M$ atteint sa valeur optimale, $I=f(b) p_{0}$ devrait tendre vers la valeur théorique. Or l'intensité expérimentale décroît d'autant plus rapidement, à $p_{0}$ donné, que $p_{1}$ est plus grand. Nous pensons que les molécules du gaz résiduel de la chambre source entrent en collision avec les molécules du faisceau ce qui rejoint l'interprétation donnée par Anderson, Andres et Fenn [4].

4.1.2 Apparition d'un maximum maximorum. Pour l'azote, comme pour la plupart des gaz, il apparaît un maximum maximorum d'intensité. Les valeurs de $p_{0}, b$ et $I$ sont d'autant plus grandes que $p_{0}$ est plus petit. Ce phénomène ne peut être dû qu'à une influence moins grande des collisions entre les molécules du gaz résiduel de la chambre source et celles du faisceau. Cette interprétation est confirmée par l'étude 
de faisceaux de gaz condensables à la température de l'air liquide tel que le propane (Fig. 9) pour lequel la pression $p_{1}$ est restée constante quel que soit $p_{0}$ et où aucun maximum maximorum n'est apparu.

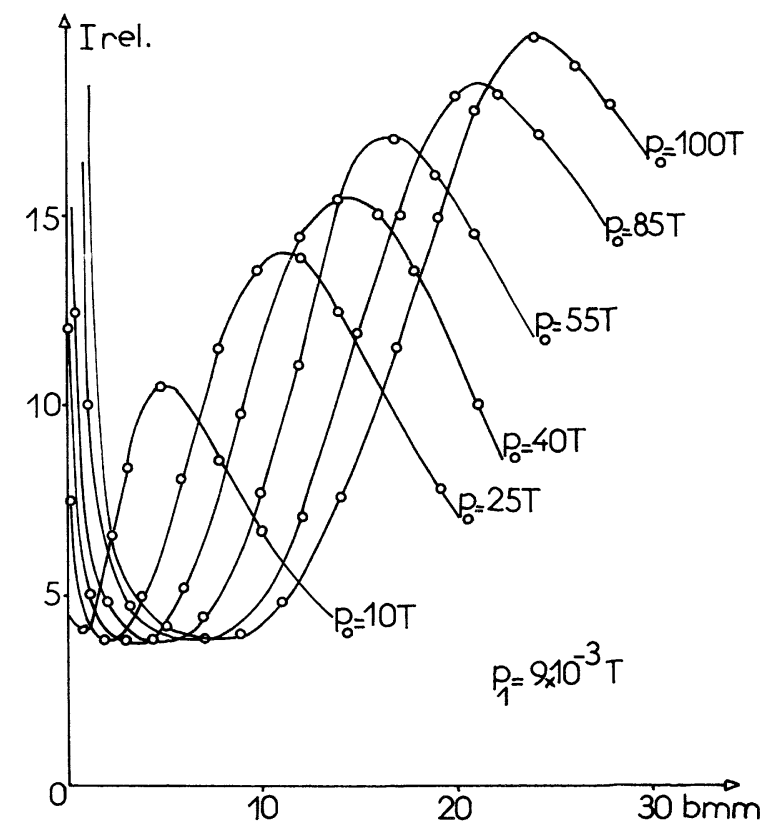

FIG. 9. - Intensité relative d'un faisceau de propane en fonction de la distance $b$ pour différentes valeurs de $p_{0}(z=9 \mathrm{~cm}$, $c=37 \mathrm{~mm}$ ).

4.2 Distribution radiale des intensités. - Pour confirmer les interprétations du paragraphe précédent, nous avons étudié les distributions des intensités pour différents gaz en faisant subir à la jauge détectrice, placée à $z=13 \mathrm{~cm}$ du collimateur, des translations dans le plan xoy suivant les directions ox et $o y$. La distance éplucheur-collimateur $c$ a été fixée à $28 \mathrm{~mm}$. L'azote est représentatif de l'ensemble des gaz.

4.2.1 Direction ox. - Nous donnons sur la figure 10, pour l'azote, les distributions obtenues pour des valeurs $b$ correspondant d'une part au régime continu, d'autre part au régime moléculaire. Pour $p_{0}=10$ torr, $b=0,15 \mathrm{~mm}$ la largeur à mi-intensité est très grande ce qui confirme la nature continue du flux. Ensuite jusqu'au maximum des courbes $I=f(b) p_{0}$ la largeur à mi-intensité diminue très nettement $[\Delta x=1,82 \mathrm{~mm}$ pour $b=1,25 \mathrm{~mm}$ et $\Delta x=1,1 \mathrm{~mm}$ pour $b=3,25 \mathrm{~mm}$ ]. Au-delà, la diminution de $\Delta x$ est très faible. Ceci est en accord avec la nature moléculaire du flux. $\Delta x$, au maximum, est inférieure à la largeur suivant $o x$ du faisceau défini par une source ponctuelle placée au centre de l'éplucheur et s'appuyant sur les bords du collimateur. Le faisceau est donc bien dirigé [11].

4.2.2 Direction oy. - La distribution suivant cet axe est donnée sur la figure 11. Au maximum, c'est-à-

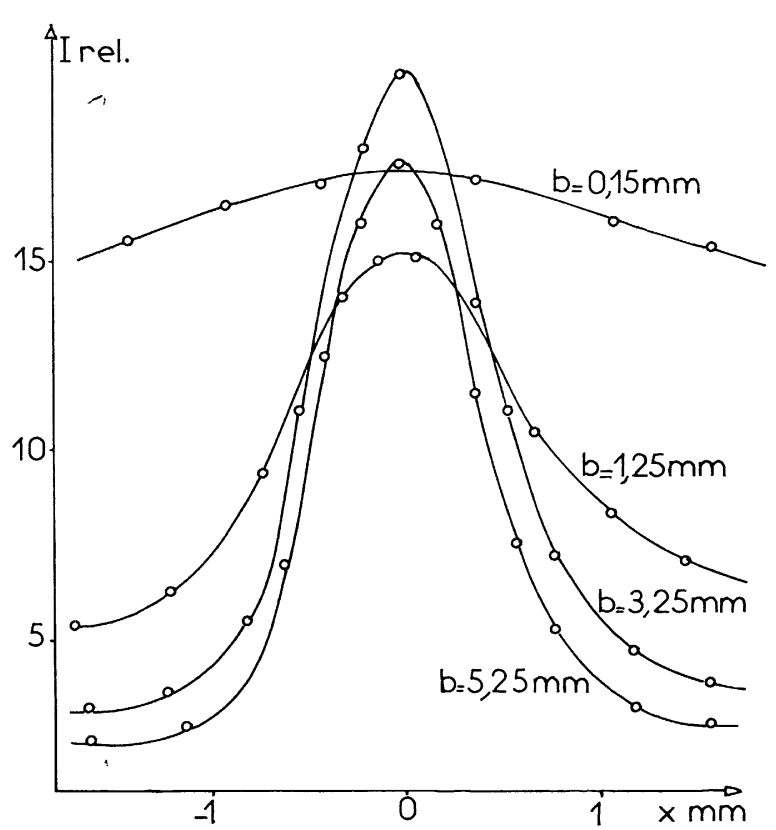

FIg. 10. - Distribution en intensité dans la direction Ox pour l'azote. $z=13 \mathrm{~cm} c=28 \mathrm{~mm}, p_{0}=10$ torr.

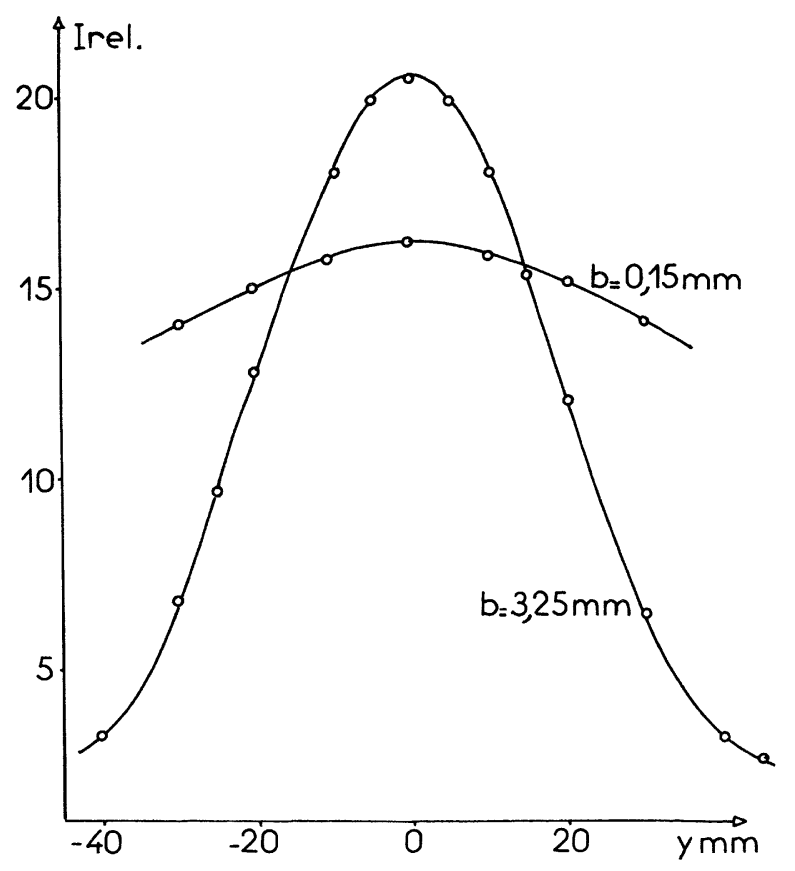

FIG. 11. - Distribution en intensité dans la direction Oy pour l'azote. $z=13 \mathrm{~cm}, c=28 \mathrm{~mm}, p_{0}=10$ torr.

dire pour $b=3,25 \mathrm{~mm}, p_{0}=10$ torr la largeur à mi-intensité $\Delta y$ est égale à $43 \mathrm{~mm}$. Cette valeur ${ }^{7}$ est très inférieure à la largeur suivant $o y$ du faisceau défini ci-dessus. Le flux est donc bien collimaté à la sortie de l'éplucheur. Nous pensons que ceci prouve qu'il est supersonique. Ce dernier point a été confirmé par une étude des distributions d'intensité dans la direction $o x$, directement à la sortie de l'éplucheur.

Au maximum de chaque courbe $I=f(b) p_{0}$, le $\mathrm{e}^{-}$flux 
est moléculaire, les perturbations à l'entrée de l'éplucheur et l'atténuation par les molécules du gaz résiduel de la chambre source sont minimales.

4.3 Calcul DU NOMbre DE MACH. - Nous avons obtenu dans la partie théorique une relation générale (4) permettant de calculer $M_{t}$. Ne connaissant pas $A$, qui dépend de $\varepsilon$, et ayant une incertitude sur $\alpha$ à cause de l'approximation faite, nous avons utilisé nos résultats expérimentaux pour calculer ces coefficients.

A la transition du flux continu en flux moléculaire, c'est-à-dire au minimum de la courbe $I=f(b) p_{0}$ le nombre de Mach $M_{\mathrm{I} \rightarrow \text { II }}$ donné par la théorie du flux continu est égal à $M_{t}$

$$
M_{I \rightarrow I I}=M_{t}=A \cdot K n_{0}^{\alpha} .
$$

A l'aide des valeurs expérimentales $b_{\text {min }}$ relevées sur les courbes, nous avons déterminé les coefficients $A$ et $\alpha$. Nous obtenons ainsi pour l'azote

$$
M_{t}=1,39 \cdot K n_{0}^{-0,36} \text {. }
$$

4.4 CAlCUl DE L'INTENSITÉ. - Nous avons produit un faisceau de four d'azote en utilisant la chambre source comme four (le gaz étant à la température ambiante et sa pression variant de $10^{-2}$ à $10^{-1}$ torr), l'éplucheur comme fente effusive et le collimateur comme fente de définition. Après avoir calculé l'intensité théorique de ce faisceau, nous avons étalonné le détecteur en mol. $\mathrm{srd}^{-1} \mathrm{~s}^{-1}$. (Le détecteur étant à $z=9 \mathrm{~cm}$.)

L'intensité expérimentale du faisceau supersonique d'azote au maximum maximorum des courbes, a alors été déterminée ; elle est de :

$$
2,1 \times 10^{18} \mathrm{~mol} \mathrm{srd}^{-1} \mathrm{~s}^{-1} .
$$

Pour les conditions du maximum maximorum, l'intensité théorique calculée à partir de la relation suivante, déduite de la relation (2),

$$
\begin{aligned}
I_{2}^{\prime}= & \frac{n_{0} S_{1} a_{0} M_{t}}{2 \pi} \times \\
& \times \frac{\left[\gamma M_{t}^{2}+3\right]}{\left[1+\frac{\gamma-1}{2} M_{t}^{2}\right]^{(\gamma+1) / 2(\gamma-1)}} \mathrm{mol} \cdot \mathrm{srd}^{-1} \cdot \mathrm{s}^{-1}
\end{aligned}
$$

est de $14,5 \times 10^{18} \mathrm{~mol} \mathrm{srd}^{-1} \mathrm{~s}^{-1}$. Le rapport de l'intensité expérimentale à l'intensité théorique, bien que faible $(14,5 \%)$, reste du même ordre de grandeur que celui obtenu par d'autres chercheurs pour des systèmes de révolution. Ce faible rapport est dû aux facteurs suivants :

- l'influence de la pression $p_{1}$ intervient avant que $K n / M$ ait atteint sa valeur optimale ;

- la réalisation de l'éplucheur étant délicate, les bords de la fente et ses angles ne sont peut-être pas géométriquement parfaits ;

- les molécules du gaz résiduel de la chambre de collimation atténuent le faisceau par collisions.
L'intensité est atténuée d'un facteur $\exp \left(-\sigma_{\mathrm{c}} \cdot n_{\mathrm{c}} \cdot c\right)$ où $\sigma_{\mathrm{c}}$ est la section efficace de collision, $n_{\mathrm{c}}$ la densité du gaz résiduel dans la chambre de collimation et $c$ la distance éplucheur-collimateur. Compte tenu de la conductance $\mathcal{C}$ de la chambre de collimation qui dépend de $c$ et de la vitesse de pompage $S_{\mathrm{c}}$ du groupe de pompage de cette chambre :

$$
\begin{aligned}
\exp ( & \left.-\sigma_{\mathrm{c}} \cdot n_{\mathrm{c}} \cdot c\right)= \\
& =\exp \left(\frac{-\sigma_{\mathrm{c}} \cdot c}{S_{\mathrm{c}}}\right) \exp \left(-\frac{2}{25} \frac{\sigma_{\mathrm{c}} \cdot L}{c^{2}}\right) \exp \left(-\frac{8}{75} \frac{\sigma_{\mathrm{c}}}{c}\right)
\end{aligned}
$$

où $L$ est le diamètre de la chambre de collimation. Cette expression passe par un maximum quand $c$ varie, ce que nous avons constaté expérimentalement (Fig. 12).

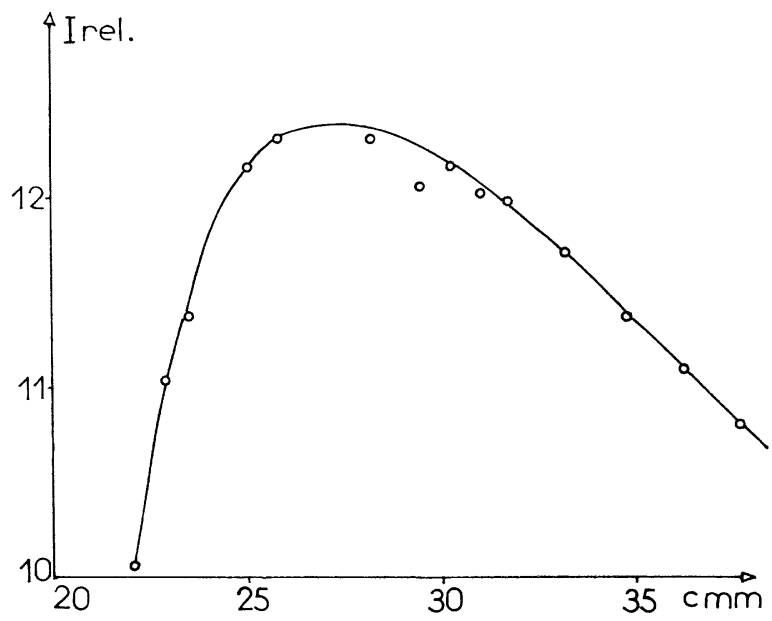

Fig. 12. - Variation de l'intensité relative en fonction de la distance $c$ pour l'azote.

5. Conclusions. - 5.1 Avantages du faisceau SUPERSONIQUE PAR RAPPORT AU FAISCEAU DE FOUR. Le faisceau supersonique obtenu présente les trois avantages suivants :

- Augmentation de l'intensité. - L'intensité par stéradian obtenue pour le faisceau supersonique d'azote est 10 fois supérieure à celle qui serait obtenue avec un faisceau de four (la fente effusive ayant les dimensions de l'éplucheur et la pression dans le four étant telle que le libre parcours moyen soit égal à la largeur de la fente).

- Rétrécissement de la distribution des vitesses. Cette distribution est de la forme :

- pour un faisceau supersonique :

$$
\left(\frac{\mathrm{d} I}{\mathrm{~d} u}\right)_{\text {sup }}=K^{\prime}\left(\frac{1}{T}\right)^{3 / 2} u^{3} \exp \left[-\frac{m}{2 R T}(U-u)^{2}\right]
$$

- pour un faisceau de four :

$$
\left(\frac{\mathrm{d} I}{\mathrm{~d} u}\right)_{\text {four }}=K^{\prime}\left(\frac{1}{T_{0}}\right)^{3 / 2} u^{3} \exp \left[-\frac{m}{2 R T_{0}} u^{2}\right]
$$

où $K^{\prime}$ a la même valeur dans (5) et (6) si les densités $n_{1}$ en amont de l'éplucheur et en amont de la fente effusive sont égales. 
La largeur de la distribution à mi-hauteur $(\Delta u)_{\text {sup }}$ a été calculée pour l'azote, pour les conditions expérimentales optimales d'obtention du faisceau (soit au maximum maximorum des courbes $\left.I=f(b) p_{0}\right)$ à partir de la relation (5)

$$
(\Delta u)_{\sup }=354 \mathrm{~m} \cdot \mathrm{s}^{-1} \text {. }
$$

La relation (6) donne, pour l'azote :

$$
(\Delta u)_{\text {four }}=504 \mathrm{~m} \cdot \mathrm{s}^{-1} \text {. }
$$

La distribution des vitesses est donc plus étroite pour le faisceau supersonique que pour un faisceau de four.

- Augmentation de l'énergie. - Pour le faisceau supersonique d'azote, dans les mêmes conditions que ci-dessus, la vitesse la plus probable est :

$$
\left(u_{\text {prob }}\right)_{\text {sup }}=766 \mathrm{~m} \cdot \mathrm{s}^{-1}
$$

alors que pour un faisceau de four

$$
\left(u_{\text {prob }}\right)_{\text {four }}=515 \mathrm{~m} \cdot \mathrm{s}^{-1} \text {. }
$$

Ceci correspond à des énergies :

$$
E_{\text {sup }}=0,086 \mathrm{eV} \quad \text { et } E_{\text {four }}=0,039 \mathrm{eV} \text {. }
$$

L'énergie obtenue pour le faisceau supersonique est supérieure à celle d'un faisceau de four mais est encore faible. Elle peut être améliorée par une augmentation de la température $T_{0}$ du gaz d'admission et par l'utilisation des mélanges de gaz [12 à 15].

5.2 Avantage SUPPlÉmENTAIRE D'UN FAISCEAU PLAN. - L'intensité optimale du faisceau supersonique plan que nous avons obtenu pour l'azote est de $2,1 \times 10^{18} \mathrm{~mol} \cdot \mathrm{srd}^{-1} \cdot \mathrm{s}^{-1}$. L'intensité totale qui passe le collimateur est de $5,3 \times 10^{15} \mathrm{~mol} . \mathrm{s}^{-1}$. Bier et Hagena [16] ont obtenu pour un faisceau supersonique de révolution une intensité de $3,6 \times 10^{18}$ mol.srd ${ }^{-1} \cdot \mathrm{s}^{-1}$ pour l'azote, l'éplucheur utilisé ayant $0,77 \mathrm{~mm}$ de diamètre c'est-à-dire même aire que le nôtre. Nous avons calculé d'après ces résultats, l'intensité totale que recevrait un collimateur de diamètre égal à la dimension caractéristique de notre collimateur plan et situé, de même, à $28 \mathrm{~mm}$ de l'éplucheur : elle serait 40 fois plus petite que la valeur que nous avons obtenue.

Une des principales applications des faisceaux supersoniques est l'étude des collisions. Dans de telles études, il est nécessaire de bien définir le point d'impact des molécules entrant en collision. Il est intéressant d'utiliser deux faisceaux croisés à $90^{\circ}$ mais nous pensons qu'il est plus avantageux d'utiliser deux faisceaux de section rectangulaire se coupant suivant leurs grandes dimensions plutôt que des faisceaux de révolution car pour une même largeur de l'impact, l'intensité totale sera plus grande donc plus facilement détectable.

\section{Bibliographie}

[1] Kantrowitz (A.) et Grey (J.), Rev. Sc. Inst., 1951, 22, 328.

[2] BecKer (E. W.) et Bier (K.), Naturforsch, 1954, 9 a, 975.

[3] Kistiakowsky (G. B.) et Slichter (W. P.), Rev. Sc. Inst., 1951, 22, 333.

[4] Anderson (J. B.), Andres (R. P.) et Fenn (J. B.), Advances in chemical Physics, 1966, X, 275-317.

[5] Bier (K.) et Schmidt (B.), Z. angew. Phys., 1961, 13, 493.

[6] Owen (P. L.) et Thornhill (C. K.), Aeronaut Research Council (U. K.), 1948, R. et M., nº 2616.

[7] Adamson (T. C.) et Nicholls (J. A.), J. Aerospace. Sci., 1959, 2, 16.

[8] Oman (B. A.), Bogan (A.), Weiser (C. H.), Li (C. H.), CAlta (V. S.), Research report, Grumannan Aircraft Eng. Co, Report R. E., 166, 1963.

[9] Hagene (M.), Pasquerault (D.), Treguier (J. P.), C. R. Acad. Sci., Paris, 1969, Série B, 268, 3654.
[10] Fenn (J. B.) et Deckers (J.), Rarefied gas dynamics, éd. Laurmann (J. A.), Acad. Press, New York, 1963, I, 497.

[11] Pasquerault (D.), Treguier (J. P.) et Hagene (M.), C. R. Acad. Sci., Paris, 1969, Série B, 269, 12.

[12] Klingelhöfer (R.) et Lohse (P.), Phys. of Fluids, 1964, 7, 379.

[13] Abuaf (N.), Anderson (J. B.), Andres (R. P.), FENN (J. B.) et Miller (D. R.), Rarefied gas dynamics, éd. Brundin (C. L.), Acad. Press, New York, 1967, II, 1317.

[14] Campargue (R.), Entropie, 1967, no 18, 55.

[15] Albright (R. G.), Peeters (J.), Bourguignon (M.), Leroy (R. L.) et Deckers (J. M.), Rarefied gas dynamics, éd. de Leeuw (J. H.), Acad. Press, New York, 1966, II, 350.

[16] Bier (K.) et Hagena (O.), Rarefied gas dynamics, éd. de Leeuw (J. H.), Acad. Press, New York, 1966, II, 260. 